\title{
Inverse Thermal Lens Effects on the Far-Field Blooming of Broad Area Laser Diodes
}

\author{
Joachim Piprek, Senior Member, IEEE
}

\begin{abstract}
High-power broad-area laser diodes often suffer from a widening of the lateral far-field with increasing current (slow-axis far-field blooming). This effect mainly originates in self-heating that generates a lateral thermal lens in the active region. Pedestal heat sinking was recently shown to mitigate the far-field blooming. In this letter, utilizing self-consistent electro-thermal optical simulations, we analyze such a far-field improvement and link it to the formation of an inverse thermal lens. At high injection current, the inverse thermal lens is shown to weaken high-order lateral modes and to even narrow the slow-axis far-field.
\end{abstract}

Index Terms-Self-focusing, semiconductor device modeling, semiconductor laser, thermo-optic effects.

$\mathbf{H}$ IGH-POWER broad-area laser diodes often suffer from a widening of the slow axis far-field with increasing current. This effect is called thermal far-field blooming, since self-heating is considered the main cause [1]. The non-uniform temperature profile inside the waveguide leads to a lateral refractive index gradient (thermal lens) that enhances the builtin index guiding of laser modes. Numerical simulation is a valuable tool to study this complex interaction of electronic, thermal, and optical processes. Previously, we published selfconsistent electro-thermal-optical simulations of the far-field blooming for a distributed feedback laser [2] and for a Fabry-Perot laser, [3] for which the simulated changes of laser power (Fig. 1), bias, wavelength, number of lateral modes, near field width, and far field angle with increasing current are all in good agreement with earlier measurements [1], [4]. Such agreement also validates the calculated internal temperature rise shown in Fig. 1. These simulations directly link the widening of the far field to a growing thermal lens which causes an increasing order of lateral lasing modes. Higherorder lateral modes are known to exhibit a wider far field.

Pedestal heat sinking was recently proposed and demonstrated to reduce the far field blooming substantially [5]. This proposal originates in the realization that the thermal lens is caused by lateral heat flow away from the active region. Thus, the reduction of that lateral heat flow by microscopic thermal path engineering promises reduced blooming and enhanced brightness [6]. The proposed pedestal heat sinking scheme

Manuscript received February 18, 2013; revised March 20, 2013; accepted March 22, 2013. Date of publication March 29, 2013; date of current version May 7, 2013. This work was supported in part by the Ferdinand Braun Institute, Berlin, Germany.

The author is with NUSOD Institute LLC, Newark, DE 19714 USA (e-mail: piprek@nusod.org).

Color versions of one or more of the figures in this letter are available online at http://ieeexplore.ieee.org.

Digital Object Identifier 10.1109/LPT.2013.2255590

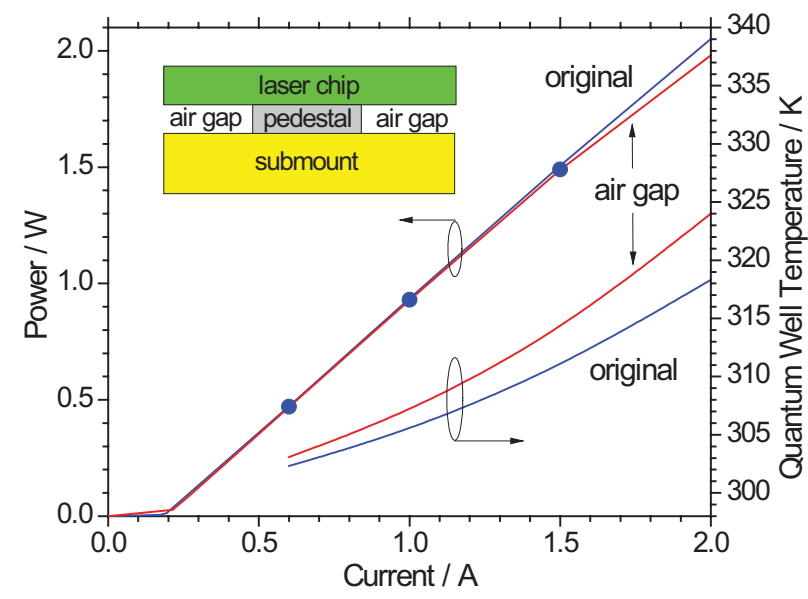

Fig. 1. Total laser power and quantum well temperature versus current before and after introducing the air gap. The dots are the originally measured power values [1]. The stage temperature is $298 \mathrm{~K}$ in both cases. The inset illustrates the pedestal heat sinking scheme, the pedestal is attached to the bottom p-contact of the laser chip.

takes this idea a step further by implementing an air gap outside the p-side contact, so that all the heat generated inside the laser is forced to flow vertically through p-contact and pedestal into the submount (see inset of Fig. 1) [5].

In the following, we use the previously studied Fabry-Perot laser [3] as an example to analyze the effects of pedestal heat sinking. The GaAs-based laser diode features a compressively strained double quantum well (QW) InGaAs/GaAsP active region that is sandwiched between $\mathrm{AlGaAs}$ waveguide and cladding layers [1], [4]. The p-contact is $50 \mu \mathrm{m}$ wide and $710 \mathrm{~nm}$ deep dry-etched trenches are located at $5 \mu \mathrm{m}$ distance on either side of the contact. The laser cavity is $1 \mathrm{~mm}$ long and the facet reflectivities are 5\% and 95\%. The relatively small size of this laser causes a large mode spacing as well as thermal lensing effects at relatively low current [1]. The laser is mounted p-side down onto a $\mathrm{CuW}$ submount. As only difference to our previously published simulations we here introduce a thin air gap to block any heat flow from the laser chip to the submount outside the p-contact (see inset of Fig. 1).

Our simulation study employs a customized version of the LASTIP laser simulation software [7] which allows for the self-consistent combination of multi-mode wave guiding, driftdiffusion of electrons and holes, heat generation, and heat flow in the transverse plane. The heat power distribution is calculated using the local densities of carriers and current. Joule heat, non-radiative recombination heat inside the quantum wells, and heat caused by modal absorption were identified as main heat sources [3]. Besides the laser chip, the 


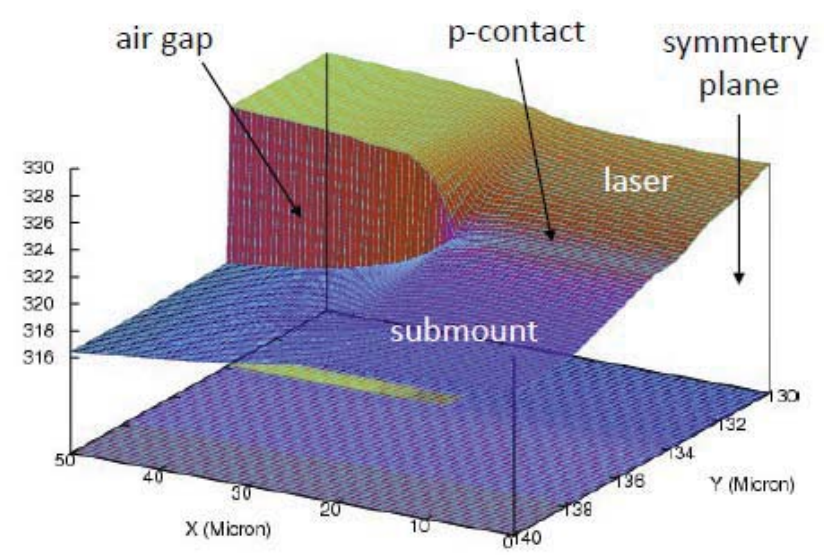

Fig. 2. Surface plot of the temperature distribution $\mathrm{T}(x, y)[\mathrm{K}]$ near the air gap at $2 \mathrm{~A}$ injection current. Based on symmetry, only half the laser is simulated.

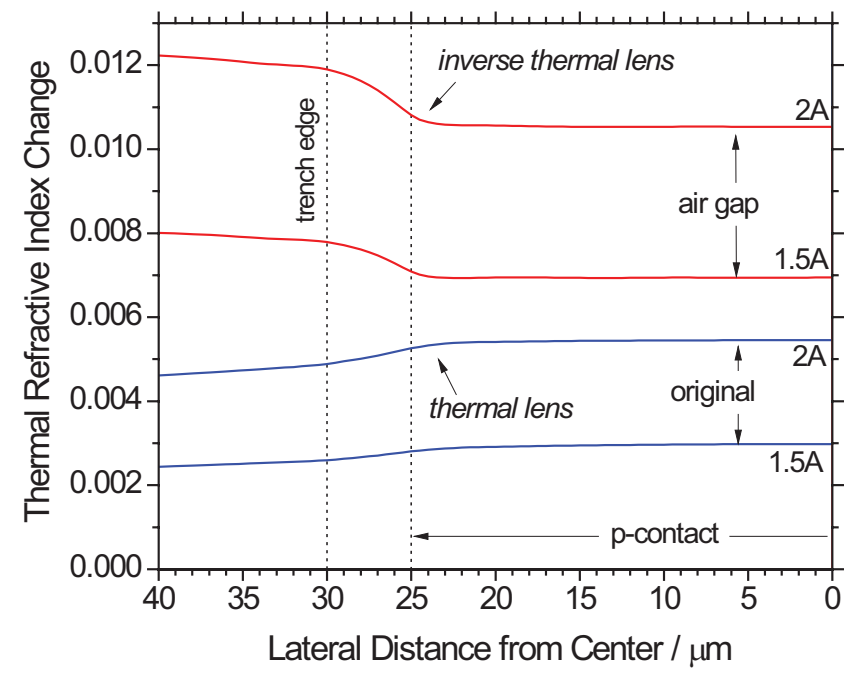

Fig. 3. Lateral profile of the thermal refractive index change in the top quantum well before and after introducing the air gap for two different currents. The effective index step built in by the trenches is 0.0017 .

simulation domain also includes the submount to accurately calculate the temperature profile $\mathrm{T}(\mathrm{x}, \mathrm{y})$. The local refractive index $\mathrm{N}(\mathrm{T}, \mathrm{x}, \mathrm{y})$ is obtained from the temperature distribution using published material parameters [8]. The average (modal) thermal index change is $\mathrm{dN} / \mathrm{dT}=3 \times 10^{-4} / \mathrm{K}$. Free-carrier absorption by holes $\left(\mathrm{k}_{p}=12 \times 10^{-18} \mathrm{~cm}^{2}\right)$ and electrons $\left(\mathrm{k}_{n}=4 \times 10^{-18} \mathrm{~cm}^{2}\right)$ is considered as well as carrier-induced index changes in the quantum wells with an anti-guiding parameter of $\mathrm{dN} / \mathrm{dn}=-10^{-20} \mathrm{~cm}^{3}$. Lateral laser modes are calculated self-consistently from the Helmholtz equation. The modal far-fields are obtained from the Fourier transform of the modal near-field at the laser facet [9]. Further details on models and parameters are given elsewhere [3], [10].

As expected, the air gap leads to a slightly increased internal temperature of the laser (Fig. 1). At 2A injection current, the quantum well temperature rises by $20 \mathrm{~K}$ before and by $26 \mathrm{~K}$ after implementation of the air gap. The lasing power is practically unchanged up to $1.5 \mathrm{~A}$ and it shows only a $3 \%$ reduction at $2 \mathrm{~A}$ (Fig. 1). The experimental investigation

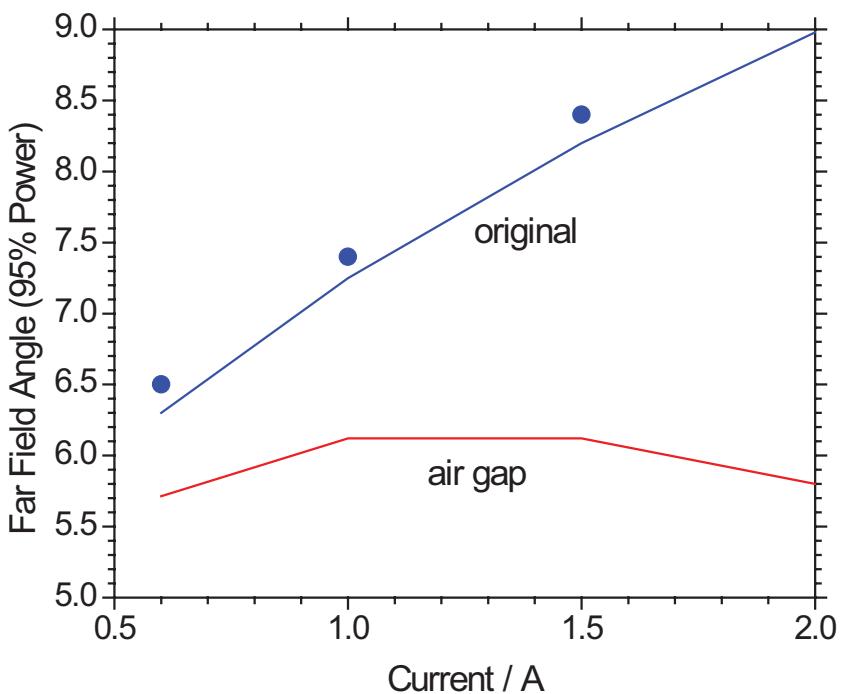

Fig. 4. Full-width slow axis far field angle versus current before and after implementation of the air gap. The dots are the originally measured values [1].

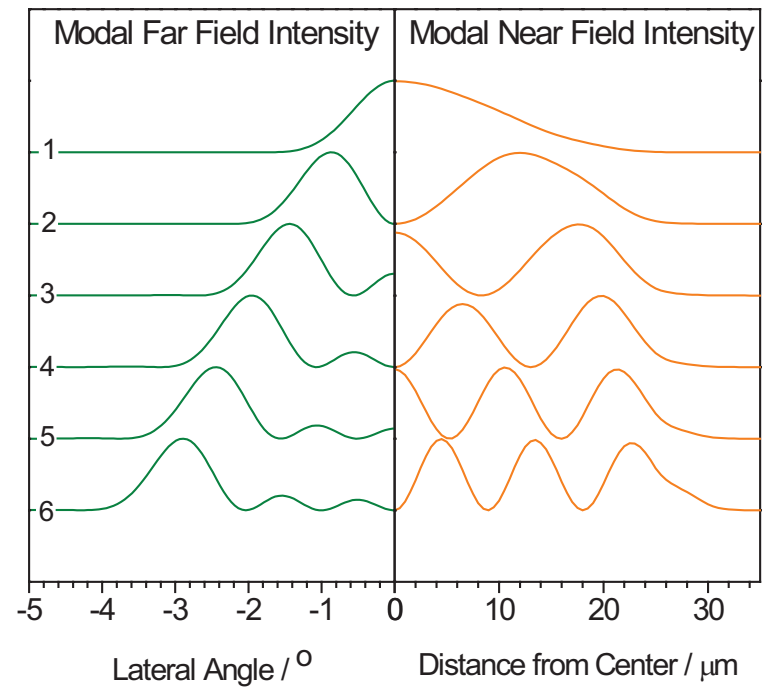

Fig. 5. Half-width lateral profiles of far field intensity and near field intensity for each individual lasing mode with air gap at $1.5 \mathrm{~A}$ (normalized). The parameter gives the lateral mode order.

of the original laser was restricted to currents up to $1.5 \mathrm{~A}$, corresponding to a current density of $3 \mathrm{kA} / \mathrm{cm}^{2}$ [1]. Fig. 2 shows the temperature distribution $\mathrm{T}(\mathrm{x}, \mathrm{y})$ near $\mathrm{p}$-contact and air gap. As intended, the air gap blocks the vertical heat flow so that all the heat flows through the p-contact, generating an inward lateral heat flow above the contact and a temperature drop towards the symmetry plane in the center of the laser. The corresponding lateral profile of the refractive index change is plotted in Fig. 3 for two different currents. In the original case, the outward heat flow away from the center creates a declining temperature and a declining refractive index. After introduction of the air gap, the heat flow is reversed so that temperature and refractive index increase with larger distance from the center. At $2 \mathrm{~A}$, this inverse thermal lens is almost as large as the effective index step of 0.0017 created by the etched 

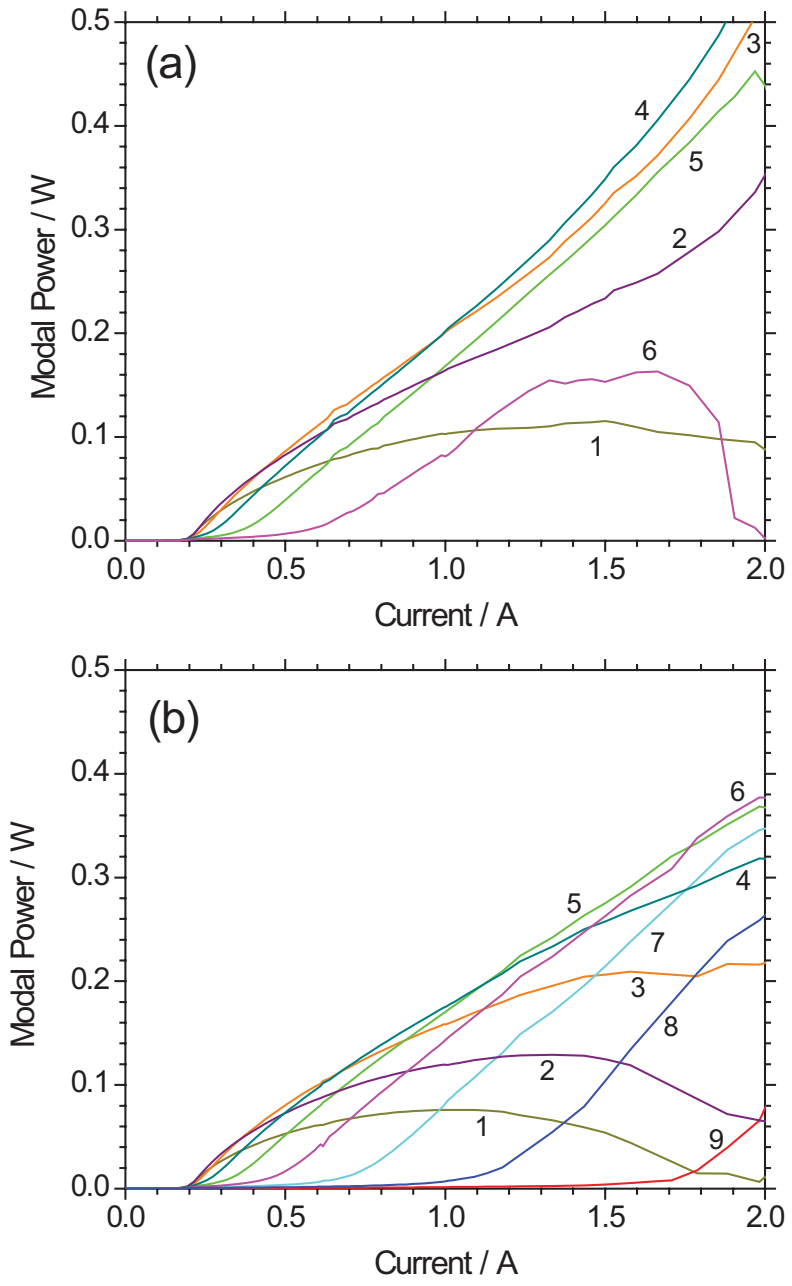

Fig. 6. Modal power versus current. The parameter gives the lateral mode order (cf. Fig. 5). (a) Air gap and (b) original.

trenches. Thus, with air gap, the self-heating weakens the builtin index guiding and is expected to also weaken higher order lateral modes (thermal anti-guiding).

Fig. 4 compares the calculated far field angles for both cases. In good agreement with the measurement, the original mounting results in a strong far-field blooming with increasing current. After implementation of the air gap, the far field angle is considerably smaller and it even shrinks between $1.5 \mathrm{~A}$ and $2 \mathrm{~A}$. This effect of the air gap on the far field can be understood by analyzing the behavior of individual lasing modes. Lateral near field and far field profiles of each mode are shown in Fig. 5 at 1.5A. A total of six lateral modes contribute to the laser power. The higher the lateral mode order, the wider is the modal far field. The power vs. current characteristic of each mode is plotted in Fig. 6a. At 0.6A, five main lateral modes are lasing, the $6^{\text {th }}$-order mode is still near threshold. At the next current step $(1 \mathrm{~A})$, the $6^{\text {th }}$ mode has fully joined the lasing action, leading to a slight increase of the far field angle in Fig. 4. The mode number does not change at $1.5 \mathrm{~A}$, so that the far field angle remains constant. However, the 6 th order mode disappears at the highest current (2A) which causes a drop in the far field angle. This disappearance of the highest-order mode is directly linked to the weakening of the built-in index guiding by the inverse thermal lens (Fig. 3).

For comparison, Fig. $6 \mathrm{~b}$ plots the modal power for the original case. The widening of the far field in Fig. 4 is mainly caused by the rising order of lateral lasing modes, each current step turns on an additional mode of higher order. Simultaneously, the fundamental mode is weakened by the increasing mode competition and by current crowding near the contact edges, which provides more gain to higher-order modes [3]. These findings are in good agreement with the experimental investigation of lateral modes [1], [4].

A common figure of merit for high power lasers is the so-called linear brightness, which is defined as lasing power divided by the half-width of the near field and the halfwidth of the far field [11]. In our case, the slow axis linear brightness at $2 \mathrm{~A}$ rises by $55 \%$ from 0.9 to $1.4 \mathrm{~W} / \mathrm{mm} * \mathrm{mrad}$ after implementation of the air gap.

In summary, we have identified the formation of an inverse thermal lens as origin of slow axis far field improvements with pedestal heat sinking.

\section{REFERENCES}

[1] P. Crump, S. Boeldicke, C. M. Schultz, H. Ekhteraei, H. Wenzel, and G. Erbert, "Experimental and theoretical analysis of the dominant lateral waveguiding mechanism in $975 \mathrm{~nm}$ high power broad area diode lasers," Semicond. Sci. Technol., vol. 27, no. 4, p. 045001, 2012.

[2] J. Piprek, "Self-consistent analysis of thermal far-field blooming of broad-area laser diodes," Opt. Quantum Electron., 2012, to be published.

[3] J. Piprek, "Self-consistent far-field blooming analysis for high-power Fabry-Perot laser diodes," Proc. SPIE, vol. 8619, p. 861910, Feb. 2013.

[4] S. Böldicke, "Characterization of lateral modes in broad-area lasers (in German)," Diploma thesis, Electrical and Computer Engineering Department, Tech. Univ. Berlin, Berlin, Germany, 2010.

[5] W. Sun, et al., "Higher brightness laser diodes with smaller slow axis divergence," Proc. SPIE, vol. 8605, p. 86050D, Feb. 2013.

[6] J. G. Bai, et al., "Mitigation of thermal lensing effect as a brightness limitation of high-power broad area diode lasers," Proc. SPIE, vol. 7953 p. 79531F, Jan. 2011.

[7] Crosslight Software Inc. (2012). LASTIP, Vancouver, BC, Canada [Online]. Available: http://www.crosslight.com

[8] S. Gehrsitz, F. K. Reinhart, C. Gourgon, N. Herres, A. Vonlanthen, and $\mathrm{H}$. Sigga, "The refractive index of $\mathrm{Al}_{x} \mathrm{Ga}_{1-x} \mathrm{As}$ below the band gap: Accurate determination and empirical modeling," J. Appl. Phys., vol. 87, no. 11, pp. 7825-7837, 2000.

[9] J. Carroll, J. Whiteaway, and D. Plump, Distributed Feedback Semiconductor Lasers. Bellingham, WA, USA: SPIE, 1998.

[10] J. Piprek, Semiconductor Optoelectronic Devices: Introduction to Physics and Simulation. San Diego, CA, USA: Academic, 2003.

[11] C. Lauer, et al., "Advances in performance and beam quality of 9xx-nm laser diodes tailored for efficient fiber coupling," Proc. SPIE, vol. 8241, p. 824111, Jan. 2012. 